\title{
Prevalence of chronic renal insufficiency in the Chinese population: A cross- sectional study
}

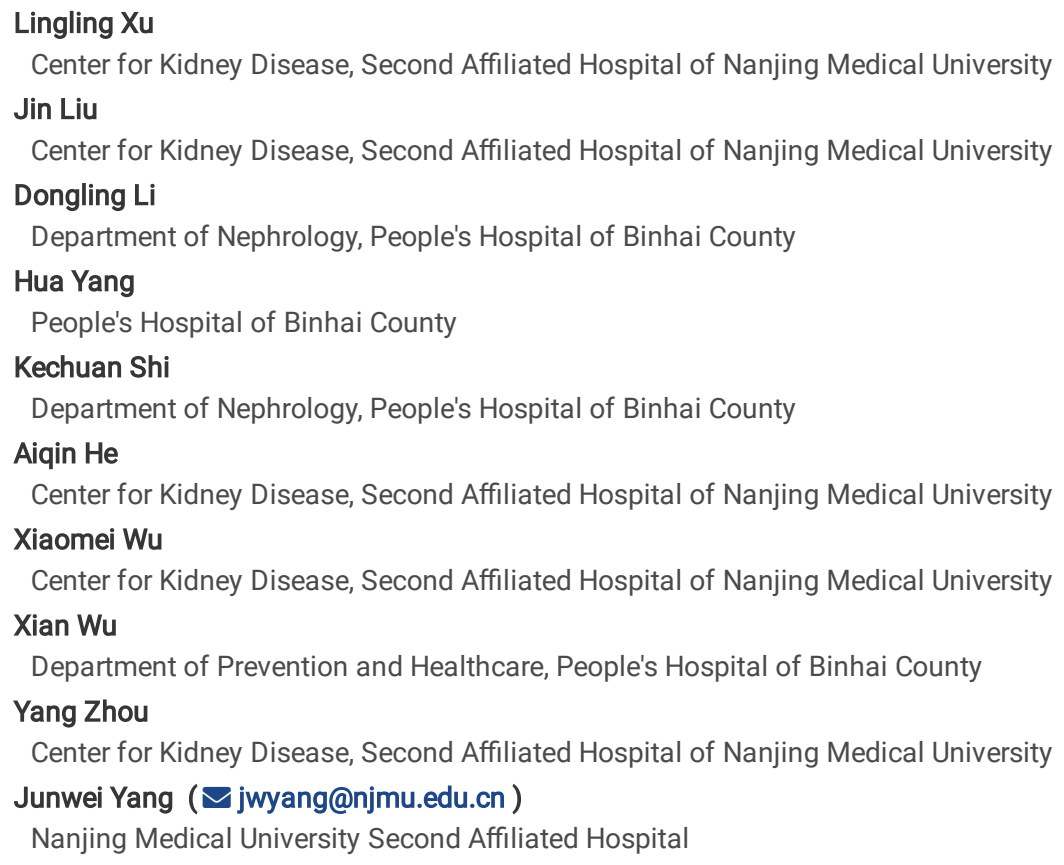

Research article

Keywords: Chronic renal insufficiency, prevalence, risk, universal health examinations

Posted Date: March 18th, 2020

DOI: https://doi.org/10.21203/rs.3.rs-17894/v1

License: (c) (i) This work is licensed under a Creative Commons Attribution 4.0 International License. Read Full License 


\section{Abstract}

Background: The prevalence of chronic kidney disease (CKD) is increasing worldwide, and it has become a global health issue with a high economic burden. Chronic renal insufficiency (CRI), the most severe form of CKD, is associated with increased risks of cardiovascular disease, premature mortality, and decreased quality of life. However, epidemiological information on Chinese patients with CRI is still lacking. This study aimed to investigate the prevalence and epidemiological features of CRI in China.

Methods: A total of 395,541 individuals aged $\geq 18$ years residing in Binhai county, China were enrolled. e estimated the prevalence of CRI adjusting for age and sex. Associations of age, sex, blood pressure, body mass index, heart rate, and fasting blood glucose and triglyceride levels with CRI were examined. Odds ratios were calculated using a binary logistic regression model. Data were obtained from the records of universal health examinations of Binhai county residents conducted from January to December 2018.

Results: The overall adjusted prevalence of CRI was 1.10\% (95\% confidence interval [CI], 1.07\%-1.13\%) in this Chinese adult population. The prevalence was $0.86 \%$ among men ( $95 \% \mathrm{Cl}, 0.82 \%-0.90 \%$ ) and $1.34 \%$ among women ( $95 \% \mathrm{Cl}, 1.29 \%-1.39 \%)$. Female sex, age (per 10-year increase), central obesity, triglyceride levels (per 118-mg/dl increase), systolic blood pressure (per 10-mmHg increase), heart rate (per 10-beats/min increase) and fasting blood glucose $\geq 100 \mathrm{mg} / \mathrm{dl}$ were found to be independent risk factors for CRI.

Conclusions: The overall prevalence of CRI in Chinese adults was $1.10 \%$. Age, female sex, central obesity, higher triglycerides, SBP, heart rate and elevated fasting glucose levels were all independent risk factors associated with decreased eGFR. Health policies to prevent CRI are urgent among this population.

\section{Introduction}

Chronic kidney disease (CKD) has been receiving increasing attention as a global public health problem. The prevalence and associated burden of CKD are rising worldwide; the global prevalence is estimated to be $8-16 \%$, with developing and undeveloped countries showing higher values [1].

Furthermore, the rapid increase in the prevalence of risk factors, such as hypertension, diabetes, and obesity, has increased the burden of CKD, making it an important socioeconomic and public health problem. China is the world's largest developing country; according to a cross-sectional survey of 47,204 Chinese adults, the nationwide prevalence of CKD was 10.8\%, while the adjusted prevalence of chronic kidney insufficiency (CRI) was $1.7 \%$ in 2012 [2]. Multiple studies have confirmed that the risks of cardiovascular disease, cardiovascular- and non-cardiovascular-related mortality, and other adverse outcomes are notably increased in individuals with lower glomerular filtration rate and albuminuria [3-5]. Screening and intervention can prevent CKD, as shown by the improvement in the incidence of end-stage kidney disease in regions where management strategies have been implemented. However, the prevalence of CRI in the general Chinese population remains unclear.

Binhai county is a representative district located in the central coastal area of the Jiangsu province in China. The universal health examination program of Binhai county residents has been launched since January 2017; this program is free and conducted by the local government. Given the availability of a large cohort of nearly half a million adults who were medically screened in a standardized manner, we are able to calculate the prevalence of CRI and evaluate its associated risk factors. The goal of this study was to provide updated estimates of CRI prevalence in the general Chinese population.

\section{Methods}

\section{Data sources and participants}

Binhai county is a district in the Jiangsu province, located in the central east coastal area of China, with an area of $1880 \mathrm{~km}^{2}$ and a permanent population of 0.96 million. The per capita income of Binhai county residents was close to the national average in 2018 . This particular region was selected because of its universal coverage of free primary care and a unique integrated electronic health information system. The system is an administrative database of general demographic characteristics and medical records. The unique feature of this system is that these databases are linked to each other by the identity card number of each individual.

The sample consisted of 398,120 participants of this standard medical screening program run by the local government and Binhai county hospital; the study period was between January and December 2018. All participants were aged $\geq 18$ years, resided in Binhai county, and had complete data for the date of birth, sex, and valid healthcare identifier. A total of 2579 participants were excluded from the study due to having an estimated glomerular filtration rate (eGFR) $>141.93 \mathrm{ml} / \mathrm{min} / 1.73 \mathrm{~m}^{2}$, which was calculated by quartile 3 plus $1.5 \times$ inter-quartile range (IQR). Finally, a total of 395,541 people were included in the study.

The study protocol was approved by the Institutional Ethical Committee of the Nanjing Medical University. All analyses followed the Strengthening the Reporting of Observational Studies in Epidemiology (STROBE) guidelines [6]. Informed consent was obtained from all study participants.

\section{Measurements And Variable Definition}

As part of the standard medical evaluation, participants underwent blood testing, body measurements, physical examination; their general demographic characteristics (sex and age) were recorded. Blood pressure, body weight, standing height, heart rate, and waist circumference were measured according to a standardized protocol. Hypertension was defined as systolic blood pressure (SBP) $\geq 140 \mathrm{mmHg}$ or diastolic blood pressure (DBP) $\geq 90 \mathrm{mmHg}$. Pre- 
hypertension was defined as SBP between 120 and $139 \mathrm{mmHg}$ and DBP between 80 and $89 \mathrm{mmHg}$ [7]. Body mass index (BMI) was calculated as weight in kilograms divided by height in meters squared $\left(\mathrm{kg} / \mathrm{m}^{2}\right)$ [8], with overweight defined as $\mathrm{BMI} \geq 24 \mathrm{~kg} / \mathrm{m}^{2}$ and obesity as $\geq 28 \mathrm{~kg} / \mathrm{m}^{2}$ by the Working Group on Obesity in China [9]. We defined central obesity based on a waist circumference greater than $80 \mathrm{~cm}$ for women or $90 \mathrm{~cm}$ for men [10]. A fasting blood sample was collected to measure serum creatinine, urea nitrogen, alanine aminotransferase, aspartate transaminase, lipids (total cholesterol and triglycerides), and plasma glucose levels. All blood samples were analyzed at the central laboratory in People's Hospital of Binhai County within 4 hours. According to the American Diabetes Association 2019 criteria, the baseline fasting blood glucose level was categorized into the following three groups: < 100, 100-125, and $\geq 126 \mathrm{mg} / \mathrm{dl}$ [11]. Serum creatinine was measured using Jaffe's kinetic method, while eGFR was calculated using the CKD Epidemiology Collaboration (CKDEPI) equation (see below) [12]. The CKD-EPI equation had less bias than the Modification of Diet in Renal Disease Study equation, especially at a GFR $\geq 60 \mathrm{ml} / \mathrm{min} / 1.73 \mathrm{~m}^{2}$ [13]. The CKD stages were categorized based on the classification system established by the National Kidney Foundation Kidney Disease Outcomes Quality Initiative guidelines. CRI (stages 3-5) was defined as decreased kidney function (eGFR $<60 \mathrm{ml} / \mathrm{min} / 1.73 \mathrm{~m}{ }^{2}$ ) in our study.

CKDEPI equations are as follows:

- eGFR $\left(\mathrm{ml} \cdot \mathrm{min}^{-1} \cdot 1.73 \mathrm{~m}^{-2}\right)=144 \times(\mathrm{Scr} / 0.7)^{-0.329} \times(0.993){ }^{\text {Age }}$ (if female, Scr $\left.\leq 0.7 \mathrm{mg} / \mathrm{dl}\right)$

- eGFR $\left(\mathrm{ml} \cdot \mathrm{min}^{-1} \cdot 1.73 \mathrm{~m}^{-2}\right)=144 \times(\mathrm{Scr} / 0.7)^{-1.209} \times(0.993)$ Age (if female, Scr $\left.>0.7 \mathrm{mg} / \mathrm{dl}\right)$

- eGFR $\left(\mathrm{ml} \cdot \mathrm{min}^{-1} \cdot 1.73 \mathrm{~m}^{-2}\right)=141 \times(\mathrm{Scr} / 0.9)^{-0.411} \times(0.993)$ Age (if male, Scr $\left.\leq 0.9 \mathrm{mg} / \mathrm{dl}\right)$

- eGFR $\left(\mathrm{ml} \cdot \mathrm{min}^{-1} \cdot 1.73 \mathrm{~m}^{-2}\right)=141 \times(\mathrm{Scr} / 0.9)^{-1.209} \times(0.993)$ Age (if male, Scr $\left.>0.9 \mathrm{mg} / \mathrm{dl}\right)$

\section{Statistical Analyses}

Prevalence were adjusted according to age and sex to represent the total population of Chinese adults by the direct method with the use of the 2010 distribution of the Chinese population [14]. Continuous data are presented as means and standard deviations. Categorical variables are presented as proportions. P values $<0.05$ were considered statistically significant, and $95 \%$ Cls were used.

We analyzed the association between $\mathrm{CRI}$ and relevant covariates with logistic regression models. The reference group in this study was composed of participants with the eGFR $\geq 60 \mathrm{ml} / \mathrm{min} / 1.73 \mathrm{~m}^{2}$. With the use of backward elimination, only significant covariates were retained in the final model. We reported multivariate adjusted odds ratios (ORs) with 95\% Cls. Covariates in the logistic regression models were age (per 10-year increment), sex, SBP (per $10-\mathrm{mmHg}$ increase), central obesity, elevated fasting glucose levels (100-125 mg/dl, $\geq 126 \mathrm{mg} / \mathrm{dl}$ ), and serum triglyceride levels (per $118-\mathrm{mg} / \mathrm{dl}$ increase). All statistical analyses were performed using SAS version 9.4 (SAS Institute, Cary, NC) and Stata version 14.1 (StataCorp, College Station, TX).

\section{Results}

\section{Participant characteristics and Prevalence of chronic renal insufficiency}

A total of 395,541 participants aged $\geq 18$ years were eligible for the study. General characteristics of the study population are presented in Table 1 . The mean age of participants in this cohort at the time of recruitment was $55.34 \pm 15.12$ years, the mean BMI was $24.51 \pm 2.92 \mathrm{~kg} / \mathrm{m}^{2}$, and $48.1 \%$ of participants were male. The mean fasting plasma glucose level was $93.24 \pm 32.31 \mathrm{mg} / \mathrm{dl}$, while the mean eGFR was $100.64 \pm 16.84 \mathrm{ml} / \mathrm{min} / 1.73 \mathrm{~m}{ }^{2}$ and the mean eGFR was higher in female participants as compared to male. 
Table 1

General characteristics of the participants.

\begin{tabular}{|c|c|c|c|c|c|c|c|c|c|c|c|}
\hline & $N(\%)$ & Age (y) & $\begin{array}{l}\text { SBP } \\
(\mathrm{mmHg})\end{array}$ & $\begin{array}{l}\text { DBP } \\
(\mathrm{mmHg})\end{array}$ & $\begin{array}{l}\text { Waist } \\
\text { Circumference } \\
\text { (cm) }\end{array}$ & $\begin{array}{l}\text { BMI } \\
\left(\mathrm{kg} / \mathrm{m}^{2}\right)\end{array}$ & $\begin{array}{l}\text { HR } \\
\text { (beats/min) }\end{array}$ & $\begin{array}{l}\text { Chol } \\
(\mathrm{mg} / \mathrm{dl})\end{array}$ & $\begin{array}{l}\text { TG } \\
(\mathrm{mg} / \mathrm{dl})\end{array}$ & $\begin{array}{l}\text { FBG } \\
(\mathrm{mg} / \mathrm{dl})\end{array}$ & $\begin{array}{l}\text { eGFR } \\
\left(\mathrm{ml} / \mathrm{min} / 1.73 \mathrm{~m}^{2}\right)\end{array}$ \\
\hline Total & 395541 & $\begin{array}{l}55.34 \\
(15.12)\end{array}$ & $\begin{array}{l}127.58 \\
(15.43)\end{array}$ & $\begin{array}{l}78.45 \\
(9.10)\end{array}$ & 81.07 (7.76) & $\begin{array}{l}24.51 \\
(2.92)\end{array}$ & $\begin{array}{l}75.61 \\
(7.73)\end{array}$ & $\begin{array}{l}194.15 \\
(38.64)\end{array}$ & $\begin{array}{l}144.89 \\
(115.69)\end{array}$ & $\begin{array}{l}93.24 \\
(32.31)\end{array}$ & $100.64(16.84)$ \\
\hline \multicolumn{12}{|l|}{ Sex } \\
\hline Male & $\begin{array}{l}190258 \\
(48.1)\end{array}$ & $\begin{array}{l}55.24 \\
(15.15)\end{array}$ & $\begin{array}{l}128.13 \\
(14.60)\end{array}$ & $\begin{array}{l}79.33 \\
(8.94)\end{array}$ & $82.74(7.27)$ & $\begin{array}{l}24.49 \\
(7.48)\end{array}$ & $\begin{array}{l}75.36 \\
(7.61)\end{array}$ & $\begin{array}{l}192.56 \\
(37.71)\end{array}$ & $\begin{array}{l}147.32 \\
(123.58)\end{array}$ & $\begin{array}{l}93.42 \\
(32.00)\end{array}$ & $100.41(16.60)$ \\
\hline Female & $\begin{array}{l}205283 \\
(51.9)\end{array}$ & $\begin{array}{l}55.43 \\
(15.08)\end{array}$ & $\begin{array}{l}127.07 \\
(16.15)\end{array}$ & $\begin{array}{l}77.64 \\
(9.17)\end{array}$ & 79.52 (7.87) & $\begin{array}{l}24.53 \\
(9.48)\end{array}$ & $\begin{array}{l}75.84 \\
(7.82)\end{array}$ & $\begin{array}{l}195.63 \\
(39.43)\end{array}$ & $\begin{array}{l}142.63 \\
(107.80)\end{array}$ & $\begin{array}{l}93.07 \\
(32.58)\end{array}$ & 100.85 (17.05) \\
\hline \multicolumn{12}{|l|}{ Age } \\
\hline $\begin{array}{l}18 \sim \\
29\end{array}$ & $\begin{array}{l}21475 \\
(5.4)\end{array}$ & $\begin{array}{l}25.65 \\
(3.03)\end{array}$ & $\begin{array}{l}118.10 \\
(9.81)\end{array}$ & $\begin{array}{l}74.02 \\
(7.16)\end{array}$ & 78.29 (8.26) & $\begin{array}{l}22.80 \\
(2.83)\end{array}$ & $\begin{array}{l}76.78 \\
(7.72)\end{array}$ & $\begin{array}{l}175.99 \\
(36.45)\end{array}$ & $\begin{array}{l}124.61 \\
(100.69)\end{array}$ & $\begin{array}{l}83.98 \\
(22.26)\end{array}$ & $123.22(13.03)$ \\
\hline $\begin{array}{l}30 \sim \\
39\end{array}$ & $\begin{array}{l}47894 \\
(12.1)\end{array}$ & $\begin{array}{l}34.53 \\
(2.85)\end{array}$ & $\begin{array}{l}119.83 \\
(11.08)\end{array}$ & $\begin{array}{l}75.22 \\
(8.09)\end{array}$ & $79.86(8.21)$ & $\begin{array}{l}23.84 \\
(2.84)\end{array}$ & $\begin{array}{l}76.40 \\
(7.51)\end{array}$ & $\begin{array}{l}181.31 \\
(36.91)\end{array}$ & $\begin{array}{l}137.42 \\
(118.45)\end{array}$ & $\begin{array}{l}86.36 \\
(25.09)\end{array}$ & $117.62(12.35)$ \\
\hline $\begin{array}{l}40 \sim \\
49\end{array}$ & $\begin{array}{l}63875 \\
(16.1)\end{array}$ & $\begin{array}{l}45.16 \\
(2.89)\end{array}$ & $\begin{array}{l}123.32 \\
(12.78)\end{array}$ & $\begin{array}{l}77.42 \\
(8.87)\end{array}$ & 80.78 (7.99) & $\begin{array}{l}24.67 \\
(2.80)\end{array}$ & $\begin{array}{l}76.20 \\
(7.39)\end{array}$ & $\begin{array}{l}188.01 \\
(36.55)\end{array}$ & $\begin{array}{l}148.49 \\
(127.61)\end{array}$ & $\begin{array}{l}90.60 \\
(29.77)\end{array}$ & 109.18 (11.21) \\
\hline $50-59$ & $\begin{array}{l}90926 \\
(23.0)\end{array}$ & $\begin{array}{l}54.19 \\
(2.77)\end{array}$ & $\begin{array}{l}127.47 \\
(14.32)\end{array}$ & $\begin{array}{l}79.21 \\
(9.12)\end{array}$ & $81.60(7.48)$ & $\begin{array}{l}24.90 \\
(2.76)\end{array}$ & $\begin{array}{l}75.32 \\
(7.38)\end{array}$ & $\begin{array}{l}197.82 \\
(37.68)\end{array}$ & $\begin{array}{l}153.75 \\
(124.67)\end{array}$ & $\begin{array}{l}94.25 \\
(33.24)\end{array}$ & $102.26(10.69)$ \\
\hline $\begin{array}{l}60 \sim \\
69\end{array}$ & $\begin{array}{l}102289 \\
(25.9)\end{array}$ & $\begin{array}{l}64.43 \\
(2.73)\end{array}$ & $\begin{array}{l}131.55 \\
(15.96)\end{array}$ & $\begin{array}{l}79.91 \\
(9.14)\end{array}$ & $81.78(7.41)$ & $\begin{array}{l}24.82 \\
(2.88)\end{array}$ & $\begin{array}{l}75.01 \\
(7.74)\end{array}$ & $\begin{array}{l}201.07 \\
(38.47)\end{array}$ & $\begin{array}{l}145.93 \\
(109.85)\end{array}$ & $\begin{array}{l}96.43 \\
(34.26)\end{array}$ & 93.71 (10.81) \\
\hline $\begin{array}{l}70 \sim \\
79\end{array}$ & $\begin{array}{l}50206 \\
(12.7)\end{array}$ & $\begin{array}{l}73.58 \\
(2.81)\end{array}$ & $\begin{array}{l}134.40 \\
(17.23)\end{array}$ & $\begin{array}{l}80.02 \\
(9.37)\end{array}$ & $81.56(7.56)$ & $\begin{array}{l}24.61 \\
(3.10)\end{array}$ & $\begin{array}{l}75.32 \\
(8.36)\end{array}$ & $\begin{array}{l}200.25 \\
(39.20)\end{array}$ & $\begin{array}{l}141.01 \\
(101.25)\end{array}$ & $\begin{array}{l}97.96 \\
(36.11)\end{array}$ & 84.80 (12.08) \\
\hline$\geq 80$ & $\begin{array}{l}18876 \\
(4.8)\end{array}$ & $\begin{array}{l}84.08 \\
(3.67)\end{array}$ & $\begin{array}{l}133.41 \\
(17.30)\end{array}$ & $\begin{array}{l}79.5 \\
(9.16)\end{array}$ & $80.58(7.49)$ & $\begin{array}{l}23.83 \\
(3.02)\end{array}$ & $\begin{array}{l}75.66 \\
(8.72)\end{array}$ & $\begin{array}{l}195.84 \\
(38.93)\end{array}$ & $\begin{array}{l}135.75 \\
(97.13)\end{array}$ & $\begin{array}{l}95.42 \\
(34.53)\end{array}$ & 74.73 (13.93) \\
\hline \multicolumn{12}{|l|}{ FBG } \\
\hline$<100$ & $\begin{array}{l}314368 \\
(79.5)\end{array}$ & $\begin{array}{l}54.30 \\
(15.32)\end{array}$ & $\begin{array}{l}126.33 \\
(14.78)\end{array}$ & $\begin{array}{l}77.93 \\
(8.91)\end{array}$ & $80.72(7.67)$ & $\begin{array}{l}24.32 \\
(2.86)\end{array}$ & $\begin{array}{l}75.37 \\
(7.55)\end{array}$ & $\begin{array}{l}191.52 \\
(37.44)\end{array}$ & $\begin{array}{l}133.39 \\
(97.22)\end{array}$ & $\begin{array}{l}82.08 \\
(9.98)\end{array}$ & 101.42 (16.68) \\
\hline $\begin{array}{l}100 \sim \\
125\end{array}$ & $\begin{array}{l}48493 \\
(12.3)\end{array}$ & $\begin{array}{l}58.83 \\
(13.66)\end{array}$ & $\begin{array}{l}132.16 \\
(16.93)\end{array}$ & $\begin{array}{l}80.45 \\
(9.66)\end{array}$ & 82.15 (7.93) & $\begin{array}{l}25.24 \\
(3.01)\end{array}$ & $\begin{array}{l}76.48 \\
(8.36)\end{array}$ & $\begin{array}{l}202.72 \\
(39.88)\end{array}$ & $\begin{array}{l}174.68 \\
(144.63)\end{array}$ & $\begin{array}{l}108.74 \\
(6.83)\end{array}$ & 97.07 (16.33) \\
\hline$\geq 126$ & $\begin{array}{l}32210 \\
(8.1)\end{array}$ & $\begin{array}{l}60.19 \\
(13.33)\end{array}$ & $\begin{array}{l}132.88 \\
(16.84)\end{array}$ & $\begin{array}{l}80.58 \\
(9.37)\end{array}$ & $82.88(7.96)$ & $\begin{array}{l}25.35 \\
(3.04)\end{array}$ & $\begin{array}{l}76.60 \\
(8.22)\end{array}$ & $\begin{array}{l}206.87 \\
(43.42)\end{array}$ & $\begin{array}{l}211.52 \\
(182.12)\end{array}$ & $\begin{array}{l}178.82 \\
(53.45)\end{array}$ & 98.32 (18.07) \\
\hline \multicolumn{12}{|l|}{ BMI } \\
\hline$<18.5$ & $\begin{array}{l}2840 \\
(0.7)\end{array}$ & $\begin{array}{l}52.62 \\
(21.74)\end{array}$ & $\begin{array}{l}120.27 \\
(17.12)\end{array}$ & $\begin{array}{l}73.85 \\
(9.73)\end{array}$ & 72.10 (7.57) & $\begin{array}{l}17.21 \\
(0.75)\end{array}$ & $\begin{array}{l}77.36 \\
(11.34)\end{array}$ & $\begin{array}{l}178.27 \\
(36.32)\end{array}$ & $\begin{array}{l}92.44 \\
(59.08)\end{array}$ & $\begin{array}{l}85.61 \\
(24.44)\end{array}$ & $103.92(21.07)$ \\
\hline $\begin{array}{l}18.5 \sim ~ \\
23.9\end{array}$ & $\begin{array}{l}177108 \\
(44.8)\end{array}$ & $\begin{array}{l}53.97 \\
(16.45)\end{array}$ & $\begin{array}{l}124.04 \\
(13.76)\end{array}$ & $\begin{array}{l}76.52 \\
(8.34)\end{array}$ & $78.24(6.60)$ & $\begin{array}{l}22.20 \\
(1.36)\end{array}$ & $\begin{array}{l}75.54 \\
(7.60)\end{array}$ & $\begin{array}{l}190.06 \\
(38.08)\end{array}$ & $\begin{array}{l}126.70 \\
(101.18)\end{array}$ & $\begin{array}{l}90.09 \\
(30.12)\end{array}$ & 102.05 (17.58) \\
\hline $\begin{array}{l}24 \sim \\
27.9\end{array}$ & $\begin{array}{l}166574 \\
(42.1)\end{array}$ & $\begin{array}{l}56.40 \\
(13.68)\end{array}$ & $\begin{array}{l}129.11 \\
(15.02)\end{array}$ & $\begin{array}{l}79.41 \\
(8.84)\end{array}$ & $82.28(6.70)$ & $\begin{array}{l}25.63 \\
(1.12)\end{array}$ & $\begin{array}{l}75.53 \\
(7.49)\end{array}$ & $\begin{array}{c}196.73 \\
(38.51)\end{array}$ & $\begin{array}{l}154.78 \\
(120.61)\end{array}$ & $\begin{array}{l}94.59 \\
(33.07)\end{array}$ & 99.64 (15.95) \\
\hline$\geq 28$ & $\begin{array}{l}44640 \\
(11.3)\end{array}$ & $\begin{array}{l}57.27 \\
(13.26)\end{array}$ & $\begin{array}{l}135.78 \\
(18.22)\end{array}$ & $\begin{array}{l}82.49 \\
(10.48)\end{array}$ & 87.55 (8.94) & $\begin{array}{l}29.99 \\
(1.84)\end{array}$ & $\begin{array}{l}75.88 \\
(8.56)\end{array}$ & $\begin{array}{l}201.36 \\
(39.28)\end{array}$ & $\begin{array}{l}179.71 \\
(134.84)\end{array}$ & $\begin{array}{l}100.54 \\
(36.04)\end{array}$ & 98.47 (16.09) \\
\hline \multicolumn{12}{|l|}{ HTN } \\
\hline $\begin{array}{l}\text { Non- } \\
\text { HTN }\end{array}$ & $\begin{array}{l}80595 \\
(20.4)\end{array}$ & $\begin{array}{l}48.01 \\
(15.25)\end{array}$ & $\begin{array}{l}110.46 \\
(6.44)\end{array}$ & $\begin{array}{l}69.90 \\
(5.77)\end{array}$ & 78.95 (7.92) & $\begin{array}{l}23.47 \\
(2.82)\end{array}$ & $\begin{array}{l}74.88 \\
(7.58)\end{array}$ & $\begin{array}{l}186.50 \\
(37.54)\end{array}$ & $\begin{array}{l}128.05 \\
(101.42)\end{array}$ & $\begin{array}{l}87.47 \\
(26.25)\end{array}$ & 106.88 (16.28) \\
\hline $\begin{array}{l}\text { Pre- } \\
\text { HTN }\end{array}$ & $\begin{array}{l}235111 \\
(59.4)\end{array}$ & $\begin{array}{l}55.48 \\
(14.80)\end{array}$ & $\begin{array}{l}125.84 \\
(6.26)\end{array}$ & $\begin{array}{l}77.90 \\
(5.87)\end{array}$ & $81.40(7.16)$ & $\begin{array}{l}24.42 \\
(2.65)\end{array}$ & $\begin{array}{l}75.59 \\
(6.76)\end{array}$ & $\begin{array}{l}194.31 \\
(38.37)\end{array}$ & $\begin{array}{l}145.15 \\
(115.73)\end{array}$ & $\begin{array}{l}92.45 \\
(32.17)\end{array}$ & $100.73(16.47)$ \\
\hline HTN & $\begin{array}{l}78812 \\
(19.9)\end{array}$ & $\begin{array}{l}62.36 \\
(12.19)\end{array}$ & $\begin{array}{l}150.30 \\
(14.41)\end{array}$ & $\begin{array}{l}88.85 \\
(9.69)\end{array}$ & 82.24 (8.81) & $\begin{array}{l}25.86 \\
(3.26)\end{array}$ & $\begin{array}{l}76.39 \\
(10.08)\end{array}$ & $\begin{array}{l}201.48 \\
(39.06)\end{array}$ & $\begin{array}{l}161.17 \\
(126.09)\end{array}$ & $\begin{array}{l}101.43 \\
(36.40)\end{array}$ & $94.02(15.90)$ \\
\hline
\end{tabular}

Data are mean (SD) or number (\%).

Abbreviation: BMI, body mass index, which is calculated as weight in kilograms divided by height in meters squared; HTN, hypertension; FBG, fasting blood glucose; SBP, Systolic blood pressure; DBP, diastolic blood pressure; HR, heart rate; TG, triglyceride; eGFR, estimated glomerular filtration rate.

SI conversion factors: To convert plasma glucose to $\mathrm{mmol} / \mathrm{L}$, multiply by 0.0555 ; total cholesterol to $\mathrm{mmol} / \mathrm{L}$ multiply by 0.0259 ; and triglycerides to $\mathrm{mmol} / \mathrm{L}$, multiply by 0.0113 . 


\begin{tabular}{|c|c|c|c|c|c|c|c|c|c|c|c|}
\hline & $N(\%)$ & Age (y) & $\begin{array}{l}\text { SBP } \\
(\mathrm{mmHg})\end{array}$ & $\begin{array}{l}\text { DBP } \\
(\mathrm{mmHg})\end{array}$ & $\begin{array}{l}\text { Waist } \\
\text { Circumference } \\
\text { (cm) }\end{array}$ & $\begin{array}{l}\text { BMI } \\
\left(\mathrm{kg} / \mathrm{m}^{2}\right)\end{array}$ & $\begin{array}{l}\text { HR } \\
\text { (beats/min) }\end{array}$ & $\begin{array}{l}\text { Chol } \\
\text { (mg/dl) }\end{array}$ & $\begin{array}{l}\text { TG } \\
(\mathrm{mg} / \mathrm{dl})\end{array}$ & $\begin{array}{l}\text { FBG } \\
(\mathrm{mg} / \mathrm{dl})\end{array}$ & $\begin{array}{l}\text { eGFR } \\
\left(\mathrm{ml} / \mathrm{min} / 1.73 \mathrm{~m}^{2}\right)\end{array}$ \\
\hline \multicolumn{12}{|c|}{ Central obesity } \\
\hline NO & $\begin{array}{l}255081 \\
(64.5)\end{array}$ & $\begin{array}{l}54.53 \\
(15.54)\end{array}$ & $\begin{array}{l}126.61 \\
(15.00)\end{array}$ & $\begin{array}{l}78.04 \\
(8.89)\end{array}$ & $77.93(6.63)$ & $\begin{array}{l}23.82 \\
(2.58)\end{array}$ & $\begin{array}{l}75.69 \\
(7.84)\end{array}$ & $\begin{array}{l}192.02 \\
(38.04)\end{array}$ & $\begin{array}{l}138.39 \\
(112.18)\end{array}$ & $\begin{array}{l}91.89 \\
(30.74)\end{array}$ & $101.39(16.88)$ \\
\hline YES & $\begin{array}{l}139676 \\
(35.3)\end{array}$ & $\begin{array}{l}56.81 \\
(14.20)\end{array}$ & $\begin{array}{l}129.35 \\
(16.02)\end{array}$ & $\begin{array}{l}79.20 \\
(9.43)\end{array}$ & $86.80(6.23)$ & $\begin{array}{l}25.80 \\
(3.07)\end{array}$ & $\begin{array}{l}75.46 \\
(7.50)\end{array}$ & $\begin{array}{l}198.03 \\
(39.42)\end{array}$ & $\begin{array}{l}156.69 \\
(120.89)\end{array}$ & $\begin{array}{l}95.67 \\
(34.83)\end{array}$ & $99.27(16.66)$ \\
\hline \multicolumn{12}{|c|}{ Data are mean (SD) or number (\%). } \\
\hline \multicolumn{12}{|c|}{$\begin{array}{l}\text { Abbreviation: BMI, body mass index, which is calculated as weight in kilograms divided by height in meters squared; HTN, hypertension; FBG, fasting } \\
\text { blood glucose; SBP, Systolic blood pressure; DBP, diastolic blood pressure; HR, heart rate; TG, triglyceride; eGFR, estimated glomerular filtration rate. }\end{array}$} \\
\hline
\end{tabular}

The mean fasting glucose level, waist circumference, serum triglyceride level, SBP, and DBP were significantly higher in male participants, whereas the cholesterol level, BMI, eGFR, and heart rate were lower in male participants.

SBP, DBP, waist circumference, BMI, triglycerides, cholesterol level, and fasting glucose level all started by increasing and then decreased with the increase in age. Conversely, eGFR decreased significantly with increasing age, BMI, and fasting glucose level. Furthermore, the mean eGFR was lower in participants with hypertension and central obesity.

In addition, the prevalence rates of hypertension and pre-hypertension in this population were $19.9 \%$ and $59.4 \%$, respectively. The percentages of people with overweight (BMI $24 \sim 27.9 \mathrm{~kg} / \mathrm{m}^{2}$ ) and obesity (BMI $\geq 28 \mathrm{~kg} / \mathrm{m}^{2}$ ) in our study were $42.1 \%$ and $11.3 \%$, respectively. According to the ADA $2019 \mathrm{criteria}, 20.4 \%$ of the population had elevated fasting blood glucose levels.

A total of 8065 (2.04\%) participants had decreased eGFR ( $\left.<60 \mathrm{ml} / \mathrm{min} / 1.73 \mathrm{~m}^{2}\right)$, including $3398 \mathrm{men}$ and 4667 women. The age- and sex-standardized overall prevalence of $\mathrm{CRI}$ in adults was $1.10 \%(95 \% \mathrm{Cl}, 1.07 \%-1.13 \%)$, with a rate of $1.34 \%(95 \% \mathrm{Cl}, 1.29 \%-1.39 \%)$ in women and $0.86 \%(95 \% \mathrm{Cl}, 0.82 \%-0.90 \%)$ in men (Fig. 1).

\section{Risk Assessment}

To characterize the risk factors for CKD, anthropometric measurements and biochemical indices were compared between subjects with and without decreased eGFR (Table 2). Participants with CRI were older, more likely to be female, and had higher BMI, waist circumference, heart rate, triglycerides, cholesterol levels, SBP and fasting glucose levels (all with $\mathrm{p}<0.001$ ). The prevalence of hypertension was $34.36 \%$ in the CRI group and $19.68 \%$ in the normal eGFR group. The proportion of participants with elevated blood glucose levels was higher in the group with decreased renal function: $16.42 \%$, blood glucose $100-125 \mathrm{mg} / \mathrm{dl}$ and $14.87 \%$, blood glucose $\geq 126 \mathrm{mg} / \mathrm{dl}$. 
Table 2

Characteristics of participants according to the renal function.

\begin{tabular}{|c|c|c|c|}
\hline Factor & $\begin{array}{l}\text { Participants with eGFR }<60 \mathrm{ml} / \mathrm{min} / 1.73 \mathrm{~m}^{2}(\mathrm{n}= \\
8065)\end{array}$ & $\begin{array}{l}\text { Participants with eGFR } \geq 60 \mathrm{ml} / \mathrm{min} / 1.73 \mathrm{~m}^{2}(\mathrm{n}= \\
387476)\end{array}$ & $\begin{array}{l}\mathrm{p} \\
\text { Value }\end{array}$ \\
\hline Male, $\mathrm{n}(\%)$ & $3389(42.02)$ & $186869(48.23)$ & $<0.001$ \\
\hline Age (y) & 72.08 (13.87) & $54.99(14.94)$ & $<0.001$ \\
\hline Waste circumference (cm) & $81.79(7.68)$ & $81.05(7.76)$ & $<0.001$ \\
\hline BMI $\left(\mathrm{kg} / \mathrm{m}^{2}\right)$ & $24.64(3.10)$ & $24.51(2.92)$ & $<0.001$ \\
\hline Heart Rate (beat/min) & $76.01(9.22)$ & $75.60(7.69)$ & $<0.001$ \\
\hline $\mathrm{TG}(\mathrm{mg} / \mathrm{dl})$ & $164.38(116.17)$ & $144.48(115.65)$ & $<0.001$ \\
\hline Chol (mg/dl) & $197.02(44.13)$ & $194.09(38.52)$ & $<0.001$ \\
\hline $\mathrm{SBP}(\mathrm{mg} / \mathrm{dl})$ & $134.25(17.93)$ & $127.44(15.34)$ & $<0.001$ \\
\hline Pre-HTN, n (\%) & $4376(54.60)$ & $230735(59.70)$ & $<0.001$ \\
\hline HTN, n (\%) & $2754(34.36)$ & $76058(19.68)$ & $<0.001$ \\
\hline $\mathrm{FBG}(\mathrm{mg} / \mathrm{dl})$ & $100.96(42.06)$ & $93.08(32.05)$ & $<0.001$ \\
\hline $\begin{array}{l}\text { FBG } 100 \sim 125 \mathrm{mg} / \mathrm{dl}, \mathrm{n} \\
(\%)\end{array}$ & $1320(16.42)$ & $47173(12.19)$ & $<0.001$ \\
\hline $\mathrm{FBG}>=126 \mathrm{mg} / \mathrm{dl}, \mathrm{n}(\%)$ & 1195 (14.87) & $31015(8.01)$ & $<0.001$ \\
\hline \multicolumn{4}{|c|}{ Data are number (\%) or mean (SD). } \\
\hline
\end{tabular}

Age (per 10-year increase), female sex, central obesity, triglycerides (per 118-mg/dl increase), SBP (per 10-mmHg increase), heart rate (per 10-beats/min increase), and elevated fasting glucose levels were all independent risk factors associated with decreased eGFR (Table 3 ). Figure 2 shows the age and sexspecific prevalence of CRI. Rates of lower eGFR differed significantly by age $(p<0.001)$, with the prevalence of lower eGFR increasing with age, especially among people aged $\geq 60$ years. As compared with those aged below 30 years, the risk of CRI was markedly increased in people aged over 60 years with an odd ratio of $4.52,14.30$ and 51.45 for $60-69,70-79$ and over 80 years old respectively (Supplementary figure S1).

Table 3

Factors associated with decrease of eGFR.

\begin{tabular}{|c|c|c|}
\hline Risk Factor & eGFR $<60 \mathrm{ml} / \mathrm{min}$ per $1.73 \mathrm{~m}^{2}$ & P Value \\
\hline Female sex & $1.142(1.083 \sim 1.203)$ & $<0.001$ \\
\hline Age per increase of $10 \mathrm{yrs}$ & $2.449(2.402 \sim 2.497)$ & $<0.001$ \\
\hline Central obesity & $1.126(1.068 \sim 1.187)$ & $<0.001$ \\
\hline Triglycerides, per increase of $118 \mathrm{mg} / \mathrm{dl}$ & $1.140(1.119 \sim 1.162)$ & $<0.001$ \\
\hline SBP, per increase of $10 \mathrm{mmHg}$ & $1.062(1.048 \sim 1.076)$ & $<0.001$ \\
\hline Heart rate, per increase of 10 beats $/ \mathrm{min}$ & $1.071(1.044 \sim 1.098)$ & $<0.001$ \\
\hline FBG $100 \sim 125 \mathrm{mg} / \mathrm{dl}$ & $1.158(1.086 \sim 1.233)$ & $<0.001$ \\
\hline $\mathrm{FBG}>=126 \mathrm{mg} / \mathrm{dl}$ & $1.387(1.296 \sim 1.484)$ & $<0.001$ \\
\hline
\end{tabular}

Data are multivariable-adjusted odds ratio $(95 \% \mathrm{Cl})$. All covariables listed were included in the model simultaneously. Central obesity was defined as a waist circumference of $90 \mathrm{~cm}$ or more in men and as $80 \mathrm{~cm}$ or more in women.

Supplementary figure S1. Odds Ratio of CRI in different ages among Chinese Adults. The odds ratio for chronic renal insufficient in different ages are shown. I bars indicate $95 \%$ confidence intervals.

\section{Discussion}

Our results showed that $\mathrm{CRI}$ has become a major public health problem in the general Chinese population. Of the 395,541 participants in our study, 8,065 had lower eGFR, corresponding to a prevalence of lower eGFR of $2.04 \%$. The age- and sex-adjusted prevalence of lower eGFR was $1.10 \%$, meaning that about 11.6 million people experience CRI in China, which is a figure similar to that found in a previous national cross-sectional survey of Chinese adults in 2012 , in which Zhang et al. reported that the adjusted prevalence of CRI in east China was also 1.1\% [2]. 
In 2009, China launched a major healthcare reform plan that pledged to provide all citizens with equal access to basic health care with reasonable quality and financial risk protection [15]. Although accomplishing nearly universal insurance coverage in such a short time is commendable, China's noncommunicable disease (NCD) control strategy needs to be strengthened to adapt to the increasing prevalence of diabetes, hypertension, and an aging population. It is recommended that a primary healthcare-based integrated system should be built in local pilot institutions.

Binhai county is located in the coastal area of East China. The average annual per capita income of residents in this area is $24,581 \mathrm{RMB}$, which is similar to the national average of 28,228 RMB. This population does not have a high immigration rate, and thus, its characteristics are representative of the native population. In addition, the local government has conducted universal free health examinations and integrated an electronic health information system for residents since January 2017. It was then gradually integrated with information on public health surveillance, population screening, disease management, hospital health information systems, and other healthcare services.

CKD has multiple causes, both inherited and acquired. Hypertension, diabetes mellitus, and obesity are important risk factors for CKD [1]. In the past two decades, hypertension and diabetes have increased rapidly in China. A previous study of 1.7 million Chinese participants indicated that $44.7 \%$ of the sample had hypertension in 2017 [16]. In 2013, another nationally representative survey of 170,287 participants identified that the estimated standardized prevalence of diabetes was $10.9 \%$ among the Chinese adult population [17]. Our study indicated that the prevalence of elevated blood glucose, elevated blood pressure, and overweight and obese BMI in these participants are high. Accordingly, our study also indicated that older age, central obesity, per 118$\mathrm{mg} / \mathrm{dl}$ increase in triglycerides, per 10-mmHg increase in SBP, and elevated fasting glucose levels were all independent risk factors associated with decreased eGFR. Furthermore, participants with lower eGFR had a higher prevalence of hypertension than participants without lower eGFR. In addition, more participants in the lower eGFR group had impaired fasting glucose tolerance (100-125 mg/dl) and fulfilled the diagnostic criteria for diabetes ( $\geq$ $126 \mathrm{mg} / \mathrm{dl}$ ). Moreover, there was a strong correlation between the decrease in eGFR and the increase in age. These results show an increase in the burden of NCDs on China's aging population.

This study has several limitations. First, this was a retrospective cross-sectional analysis, meaning that some information about the participants' social and demographic status, personal and family health history, lifestyle, histories of cardiovascular disease, diabetes, and hypertension, and nephrotoxic medication use was missing. Second, all covariates were obtained from single measurements; therefore, the reported prevalence of CRI, hypertension, and pre-hypertension might be biased.

In conclusion, the estimated overall prevalence of CRI in Chinese adults in 2018 was $1.10 \%$. Special attention should be paid to subjects aged 60 years and older. The increasing prevalence of diabetes and hypertension in an aging population would increase the prevalence of CRI and have a profound impact on China's social economy and public health. It is recommended that China's NCD control strategy be strengthened to meet the demands of the aging population.

\section{Abbreviations}

BMI

body mass index

CKD

chronic kidney disease

CKDEPI

chronic kidney disease epidemiology collaboration

CRI

chronic renal insufficiency

DBP

diastolic blood pressure

eGFR

estimated glomerular filtration rate

IQR

inter-quartile range

NCD

non-communicable disease

OR

odds ratio

SBP

systolic blood pressure

STROBE

strengthening the reporting of observational studies in epidemiology

\section{Declarations}

\section{Authors' Contributions}


J.Y, Y.Z. and X.W. conceived the study. L.X., J.L., D.L., H.Y, K.S., A.H. and X.W. were involved in clinical care of the participants and data acquisition. L.X. and Y.Z. were involved in analyzing the data and writing the initial draft of the manuscript., J.Y. J.L., D.L., H.Y, K.S., A.H. and X.W. were involved in editing and revision of the manuscript. All authors approved the final manuscript. J.Y. is the guarantor of this work and, as such, had full access to all the data in the study and takes responsibility for the integrity of the data and the accuracy of the data analysis.

\section{Support}

The study is supported by the grants from National Natural Science Foundation of China BE2017762 to Junwei Yang. National Natural Science Foundation of China: Key program 81530022 and General program 81873618 to Junwei Yang. Youth fund 81600526 to Yang Zhou. Youth medical talent fund QNRC2016670 to Yang Zhou.

\section{Financial disclosure}

No conflicts of interest, financial or otherwise, are declared by the authors.

\section{Acknowledgements}

The investigators are grateful to all participants and their families for their commitment to this study. We would like to thank Editage (www.editage.cn) for English language editing.

\section{References}

1. Jha V, Garcia-Garcia G, Iseki K, Li Z, Naicker S, Plattner B, Saran R, Wang AY, Yang CW. Chronic kidney disease: global dimension and perspectives. Lancet. 2013; 382:260-272.

2. Zhang L, Wang F, Wang L, Wang W, Liu B, Liu J, Chen M, He Q, Liao Y, Yu X et al. Prevalence of chronic kidney disease in China: a cross-sectional survey. Lancet. 2012; 379:815-822.

3. Hostetter TH. Chronic kidney disease predicts cardiovascular disease. The New England journal of medicine. 2004; 351:1344-1346.

4. Go AS, Chertow GM, Fan D, McCulloch CE, Hsu CY. Chronic kidney disease and the risks of death, cardiovascular events, and hospitalization. The New England journal of medicine. 2004; 351:1296-1305.

5. Fried LF, Katz R, Sarnak MJ, Shlipak MG, Chaves PH, Jenny NS, Stehman-Breen C, Gillen D, Bleyer AJ, Hirsch C et al. Kidney function as a predictor of noncardiovascular mortality. Journal of the American Society of Nephrology : JASN. 2005; 16:3728-3735.

6. von Elm E, Altman DG, Egger M, Pocock SJ, Gotzsche PC, Vandenbroucke JP, Initiative S. The Strengthening the Reporting of Observational Studies in Epidemiology (STROBE) statement: guidelines for reporting observational studies. Lancet. 2007; 370:1453-1457.

7. Wang Z, Chen Z, Zhang L, Wang X, Hao G, Zhang Z, Shao L, Tian Y, Dong Y, Zheng C et al. Status of Hypertension in China: Results From the China Hypertension Survey, 2012-2015. Circulation. 2018; 137:2344-2356.

8. Clinical Guidelines on the Identification, Evaluation, and Treatment of Overweight and Obesity in Adults-The Evidence Report. National Institutes of Health. Obesity research. 1998; 6 Suppl 2:51S-209S.

9. Zhou B, Coorperative Meta-Analysis Group Of China Obesity Task F. [Predictive values of body mass index and waist circumference to risk factors of related diseases in Chinese adult population]. Zhonghua liu xing bing xue za zhi = Zhonghua liuxingbingxue zazhi. 2002; 23:5-10.

10. Alberti KG, Zimmet P, Shaw J, Group IDFETFC. The metabolic syndrome-a new worldwide definition. Lancet. 2005; 366:1059-1062.

11. Cosentino F, Grant PJ, Aboyans V, Bailey CJ, Ceriello A, Delgado V, Federici M, Filippatos G, Grobbee DE, Hansen TB et al. 2019 ESC Guidelines on diabetes, pre-diabetes, and cardiovascular diseases developed in collaboration with the EASD. European heart journal. 2019.

12. Levey AS, Stevens LA, Schmid CH, Zhang YL, Castro AF, 3rd, Feldman HI, Kusek JW, Eggers P, Van Lente F, Greene T et al. A new equation to estimate glomerular filtration rate. Annals of internal medicine. 2009; 150:604-612.

13. Earley A, Miskulin D, Lamb EJ, Levey AS, Uhlig K. Estimating equations for glomerular filtration rate in the era of creatinine standardization: a systematic review. Annals of internal medicine. 2012; 156:785-795, W-270, W-271, W-272, W-273, W-274, W-275, W-276, W-277, W-278.

14. Tabulation on the 2010 population census of the people's republic of China [http://www.stats.gov.cn/tjsj/pcsj/rkpc/6rp/indexch.htm]

15. Yip WC, Hsiao WC, Chen W, Hu S, Ma J, Maynard A. Early appraisal of China's huge and complex health-care reforms. Lancet. 2012; 379:833-842.

16. Lu J, Lu Y, Wang X, Li X, Linderman GC, Wu C, Cheng X, Mu L, Zhang H, Liu J et al. Prevalence, awareness, treatment, and control of hypertension in China: data from 1.7 million adults in a population-based screening study (China PEACE Million Persons Project). Lancet. 2017; 390:2549-2558.

17. Wang L, Gao P, Zhang M, Huang Z, Zhang D, Deng Q, Li Y, Zhao Z, Qin X, Jin D et al. Prevalence and Ethnic Pattern of Diabetes and Prediabetes in China in 2013. Jama. 2017; 317:2515-2523.

\section{Supplementary Figure Caption}

Supplementary figure S1. Odds Ratio of CRI in different ages among Chinese Adults. The odds ratio for chronic renal insufficient in different ages are shown. I bars indicate $95 \%$ confidence intervals.

\section{Figures}


A

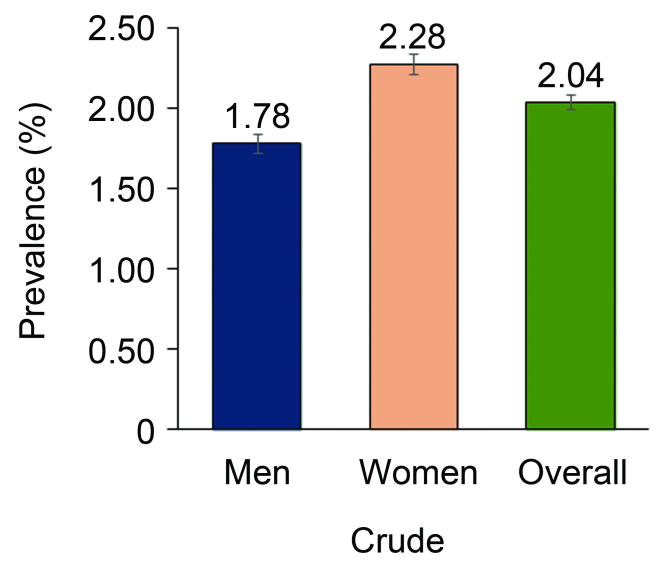

B

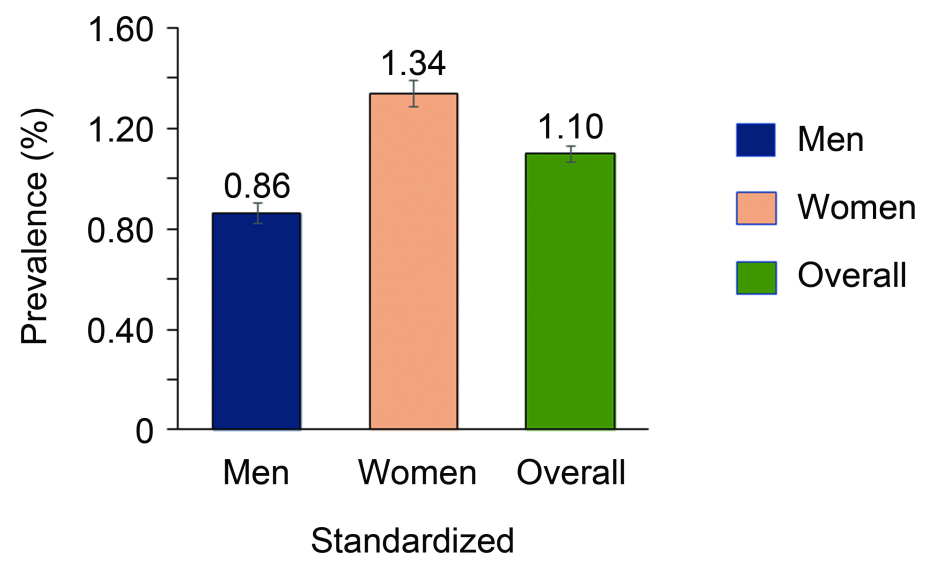

Figure 1

The crude and standardized prevalence of chronic renal insufficient among Chinese adults. The crude and age-standardized prevalence of chronic renal insufficient among men and women are shown in panels A and B, respectively. Age- and sex-standardized prevalence were calculated by the direct method with the use of data on the population distribution in China in 2010. I bars indicate $95 \%$ confidence intervals.

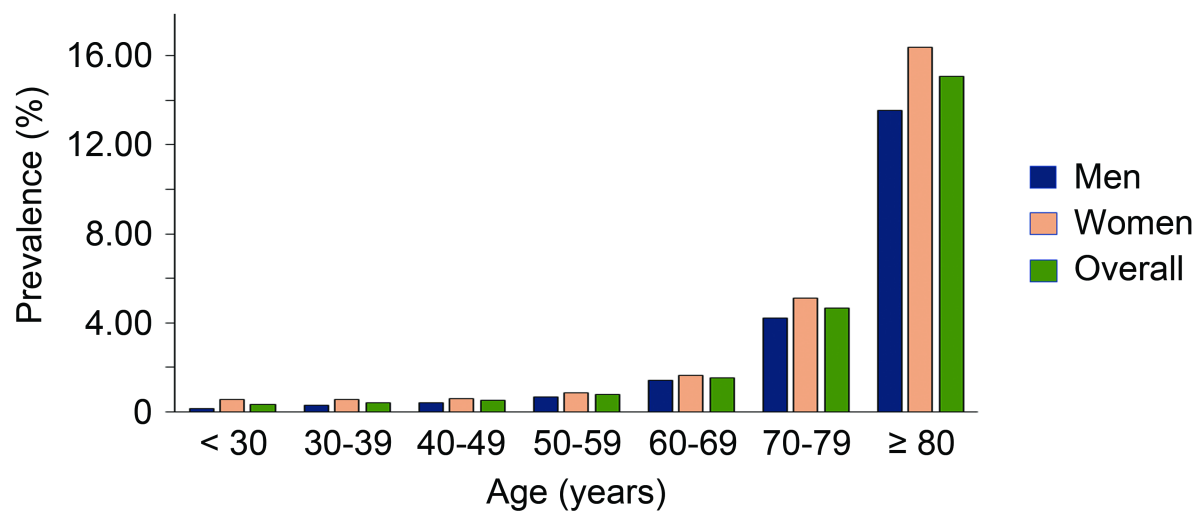

Figure 2

Age-specific prevalence of chronic renal insufficient among Chinese adults. The age-specific prevalence of chronic renal insufficient among men, women and all participants are shown.

\section{Supplementary Files}

This is a list of supplementary files associated with this preprint. Click to download.

- CRIFigureS1.tif 